\title{
Modélisation des courants de houle et du transport sédimentaire pour l'étude de stabilité de plage
}

\author{
Richard Marcer \\ Ingénieur chef de projet, PRINCIPIA R.D. \\ Nadège Robin \\ Ingénieur stagiaire, PRINCIPIA R.D.
}

\section{Résumé}

Dans le cadre du projet Eureka concernant la validation du concept d'atténuateurs de houle de type plaque comme système de protection du littoral, une chaîne de calcul couplant des modèles de houle, de courantologie et de transport sédimentaire, a été développée. Le modèle de houle de type parabolique permet de prendre en compte les effets de réfraction et de diffraction. Le transport littoral est calculé à partir de modèles de courant et de transport sédimentaire bidimensionnels qui donnent de bons résultats lorsque l'on s'intéresse aux évolutions sédimentaires à une échelle globale. Un exemple d'application de la chaîne de calcul pour l'étude de stabilité de plage est présenté.

\section{Introduction}

Les transits sédimentaires qui font qu'un site n'est jamais en équilibre dynamique constant, peuvent provoquer des érosions ou des dépots néfastes à l'équilibre naturel de ce site. L'étude du comportement des sédiments en milieu côtier est particulièrement complexe car de multiples processus instationnaires sont mis en jeu et interagissent. S'il est difficile de les représenter avec précision, il est néanmoins possible d'en mettre en évidence les mécanismes moteurs et de les modéliser moyennant un certain nombre d'hypothèses.

Le calcul du transport littoral dû aux courants engendrés par le déferlement peut être réalisé par des outils numériques bidimensionnels moyennés sur la profondeur d'eau qui permettent de donner des tendances globales d'évolution de profil de plage soumis aux effets de la houle et des courants, et d'aider à la prédiction de l'efficacité d'ouvrages de protection.

Dans la modélisation présentée ici, le modèle de transport sédimentaire qui permet de déterminer les évolutions de la bathymétrie en milieux sableux, utilise comme forçages hydrodynamiques les données d'un modèle de courantologie 2-D et d'un modèle de réfraction-diffraction de la houle. 


\section{Modèles numériques}

\subsection{Modèle de houle}

\subsubsection{Rappel des équations de base}

Le comportement de la houle à l'approche des côtes est modélisé par l'équation de Berkhoff. Cette équation traduit les équations du mouvement en théorie linéaire pour le cas de fond lentement variable. Elle s'écrit:

$$
\nabla\left(C . C_{g}, \nabla \varphi\right)+\frac{C_{g}}{C} \omega^{2} \varphi=0
$$

avec:

$C=\omega / k$ vitesse de phase

$C_{g}=\frac{C}{2}\left(1+\frac{2 k h}{\operatorname{sh}(k h)}\right)$ vitesse de groupe

$\varphi(x, y)$ potentiel horizontal

$\omega^{2}=g k t h(k h)$ relation de dispersion

L'équation (1) peut être résolue directement, par exemple, par un modèle aux éléments finis [1]. Ce type de modèle est notamment destiné à étudier les problèmes d'agitation portuaire où les effets combinés de diffraction et réflexion de la houle peuvent être importants. Pour des applications à échelle plus globale (zone littorale, plage), il est parfois préférable d'utiliser des modèles de houle plus maniables tels que le modèle parabolique décrit ci-après.

\subsubsection{Modèle parabolique}

En supposant la réfraction prépondérante devant la diffraction et la réflexion de la houle, il est possible de projeter l'équation elliptique de Berkhoff sur l'axe principal de propagation. En supposant $x$ cet axe, on montre que le potentiel obéit à l'équation:

$$
\frac{\partial \varphi}{\partial x}=\left(i k-\frac{1}{2 k C C_{g}} \frac{\partial}{\partial x}\left(k C C_{g}\right)\right)+\frac{i}{2 k C C_{g}} \frac{\partial}{\partial y}\left(C C_{g} \frac{\partial \varphi}{\partial y}\right)
$$

Cette dernière équation est l'équation de Berkhoff parabolisée qui présente une dérivée au premier ordre uniquement suivant la direction $x$ de propagation. L'avantage de cette équation parabolique est qu'elle peut être résolue par une simple méthode de différences finies en progressant ligne par ligne. Le schéma de discrétisation utilisé est celui de Cranck-Nicholson.

Le modèle parabolique, destiné à simuler la propagation de la houle du large vers la côte, prend en compte les effets de réfraction et de diffraction de la houle autour de la direction principale de propagation. Son domaine de validité se situe entre celui des 
modèles de réfraction pure de type rayon et celui des modèles de type éléments finis [1] qui prennent en compte, en plus, la réflexion de la houle.

\subsubsection{Déferlement}

Le déferlement est traité par une relation qui permet de reproduire les non-linéarités liées à ce phénomène. Les points de déferlement sont déterminés selon le critère de Battjes qui indique qu'il y a déferlement dès que $\eta \geq \gamma h$, avec $\eta$ l'amplitude de la houle, $h$ la hauteur d'eau et $\gamma$ un critère de déferlement, compris entre 0,8 et 1,2 , qui dépend de la cambrure locale de la houle. L'évolution de la hauteur de houle dans le déferlement est donnée par la formule de Stive [1]:

$$
\frac{\partial \eta}{\partial x}+\frac{\eta}{4 h} \frac{\partial h}{\partial x}=A\left(\frac{h}{g T^{2}}\right)^{\frac{1}{2}} \frac{\eta}{h}
$$

où $T$ est la période de la houle et $A$ un coefficient empirique valant 1,3 .

\subsection{Modèle de courantologie}

Le calcul du champ de vitesse induit par la houle est réalisé avec un code de calcul des équations de Saint-Venant moyennées sur la verticale. Ces équations s'écrivent:

$$
\begin{gathered}
\frac{\partial \varsigma}{\partial t}+\frac{\partial H V_{i}}{\partial x_{i}}=0 \\
\frac{\partial H V_{i}}{\partial t}+\frac{\partial}{\partial x_{i}} H V_{i} V_{j}=-g H \frac{\partial \varsigma}{\partial x_{i}}+\frac{\partial T_{i j}}{\partial x_{i}}+\tau_{i}^{b}+\tau_{i}^{\omega}+\tau_{i}^{m}
\end{gathered}
$$

avec:

$H$ la hauteur d'eau totale

$\varsigma$ l'élévation de la surface libre

$\tau_{i}^{b}$ la contrainte de frottement sur le fond

$\tau_{i}^{w}$ la contrainte de frottement sur la surface (vent)

$\tau_{i}^{m}$ les tensions de radiation

$T_{i j}$ les tensions de Reynolds

Dans ce système d'équations, le terme $\tau_{i}^{m}$ traduit l'effet de la houle sur la mise en mouvement des masses d'eau et l'établissement des courants qui agissent principalement dans la zone déferlement. Ce terme, appelé également terme moteur, se comporte comme un terme de contrainte de même type que les contraintes de frottement sur le fond et la surface. Il s'écrit: 


$$
\begin{aligned}
& \tau_{i}^{m}=\frac{-1}{\rho} \frac{\partial S_{i j}}{\partial x_{i}} \\
& S_{i j}=f(\eta, \vec{\nabla} \eta, k, \theta)
\end{aligned}
$$

La fonction $f(\eta, \nabla \eta, k, \theta)$ fait intervenir lamplitude de la houle, les gradients $d^{\prime \prime}$ amplitude, le nombre d'onde local et l'incidence de la houle qui est une fonction du gradient de la phase de l'onde dans les deux directions. Ces gradients sont calculés par différences finies sur le maillage du modèle de houle. Le couplage numérique entre les modèles de houle et de courantologie est réalisé à partir d'une procédure qui permet d'affecter, au moyen d'interpolations, les données de houle sur le maillage du modèle courantologie.

\subsection{Modèle de transport sédimentaire}

Le modèle de sédimentologie est un modèle de transport par charriage, par suspension et par saltation. Les lois de transport utilisées sont celles définies par Bijker [2] qui permet de prendre en compte les effets combinés de la houle et du courant lors de la mise en suspension et le transport des particules.

Pour délimiter les différents types de transport, on considère que les particules qui restent près du fond sont entrainées par charriage jusqu'à une limite donnée par la hauteur des rides du fond; au delà, le transport s'effectue par suspension.

Le transport par saltation concerne les particules qui se décollent et retombent successivement. Il est pris en compte dans les lois de transport par charriage et suspension.

Nous décrivons sommairement le principe de cette méthode et renvoyons à l'article de base [2] pour une description détaillée des lois de transport.

\subsubsection{Transport par charriage}

La loi de transport est de la forme:

$$
T_{c}=A e^{B}
$$

où $A$ correspond à un débit de matériau transporté et $B$ à une fonction de la contrainte de cisaillement due au courant et à la houle. Le terme exponentiel simule la mise en mouvement des particules solides, en considérant que la probabilité de mise en mouvement diminue lorsque la tension de frottement décroit.

La relation (8) fait intervenir un certain nombre de paramètres tels que la vitesse du courant, la vitesse orbitale de la houle, le diamètre des grains, la densité des grains, la vitesse de sédimentation des particules, le coefficient de Chezy et le coefficient de ride. 


\subsubsection{Transport par suspension}

Le transport par suspension est obtenu en sommant sur la colonne d'eau, à partir d'un niveau $h_{r}$ correspondant à la hauteur des rides du fond, le produit de la concentration volumétrique et de la vitesse horizontale moyenne:

$$
T_{s}=\int_{h_{T}}^{h} c(z) u(z) d z
$$

Généralement le débit solide en suspension est environ 7 à 10 fois plus grand que le débit charrié.

\subsubsection{Loi de conservation du matériau}

L'évolution des fonds est donnée par la relation:

$$
\frac{\partial z}{\partial t}+d i v \vec{T}=0
$$

où $\vec{T}=\vec{T}_{c}+\vec{T}_{s}$

Cette équation est résolue par différences finies. Le maillage est identique à celui du modèle de courantologie. Les composantes du transport sédimentaire sont calculées en chaque noeud de la grille où sont connues les caractéristiques de la houle et des composantes de vitesse du courant. Pour le calcul du terme de divergence, on utilise des différences finies avant ou arrière suivant le signe des composantes du transport, de façon à toujours considérer le point en amont du point de discrétisation. Les conditions limites portent sur la frontière d'entrée du domaine où les fonds sont supposés rester inchangés. Sur les autres frontières, le calcul est réalisé de la même manière que pour les points à l'intérieur du domaine.

La résolution de l'équation (10) permet de déterminer en chaque point la cote du fond $z$ au temps $t^{n+1}$, connaissant l'état de la bathymétrie à l'instant $t^{n}$. Lorsque l'évolution de la bathymétrie est significative, il est nécessaire de réactualiser le plan de vagues et les courants de houle induits.

\section{Résultats numériques}

L'exemple numérique présenté concerne une baie semi-circulaire de $260 \mathrm{~m}$ de rayon, avec un fond de pente régulière à $3 \%$ et une profondeur maximale de $7,5 \mathrm{~m}$ au large (figure 1). Les conditions de houle du large sont $\eta=3 m, T=10 \mathrm{~s}$ et $\theta=60^{\circ}$. Le nombre de points par longueur d'onde est fixé à 15 . Les caractéristiques du sable considéré sont le diamètre des grains $D=0,5 \mathrm{~mm}$, la vitesse de chute $W_{s}=0.08 \mathrm{~cm} / \mathrm{s}$ et la hauteur des rides du fond $h_{r}=0,15 \mathrm{~m}$.

Le champ de phase montre que le plan de vague a tendance, sous l'effet de la réfraction, à suivre la bathymétrie (figure 2).

L'allure du champ de débit solide (figure 3), identique à celui du champ de courant puisque le transit à la même direction que l'écoulement moyen, présente quelques 
caractéristiques dominantes: deux larges tourbillons contra-rotatifs de part et d'autre de la baie placés sur la barre de déferlement où les gradients d'amplitude de la houle sont les plus importants, des tourbillons secondaires dûs aux effets de diffraction de la houle sur la partie gauche du domaine, et une circulation vers le large relativement intense au centre de la baie où l'amplitude de la houle est assez régulière (figure 2).

L'évolution de la bathymétrie sur 50h d'action de la houle et des courants induits, est représentée sur la figure 4 . On note globalement deux grandes tendances: des phénomènes de sédimentation sur la partie gauche et au centre de la baie, des processus d'érosion sur la partie droite de la baie et le long de la zone centrale de la plage où les sédiments sont entrainés vers le large par le courant.

\section{Conclusions et perspectives}

Les premiers résultats numériques présentés dans cet article ont permi de valider la méthodologie de calcul de la stabilité d'une plage à partir d'un couplage des différents modèles de houle, de courant et de transport sédimentaire développés à PRINCIPIA. Des comparaisons complémentaires, d'une part avec des résultats d'autres codes pour le même cas d'étude [3] et, d'autre part avec des formules globales et des modèles physiques (plage rectiligne infinie), ont permis de conclure à la bonne fiabilité des outils numériques.

Une poursuite de la validation du modèle est actuellement en cours. Elle permettra à très court terme d'aboutir à la réalisation d'un outil finalisé capable de prédire les tendances générales de la dynamique d'une plage quelconque et d'estimer, si besoin, les configurations optimales d'ouvrages à mettre en oeuvre pour améliorer la stabilité de la plage.

\section{Références}

[1] Marcer R., Landel E., Guerin P.

"Modélisation de l'influence des atténuateurs de houle sur la protection du littoral", 3ème Journées Nationales Génie civil-Génie côtier, Sète, 2-4 Mars, 1994.

[2] Bijker E.W.

"Littoral drift as function of waves and current", Proc., XIth. Conf. Coast. Eng., London, pap. 27, 1968.

[3] Péchon $P$.

"Transport littoral par la houle", Rapport du Laboratoire National d'Hydraulique, Mai 1982.

\section{Remerciements}

Ces travaux ont été réalisés dans le cadre du projet Eureka EU394. Ils ont été financés en partie par le Ministère de la Recherche et de la Technologie. 


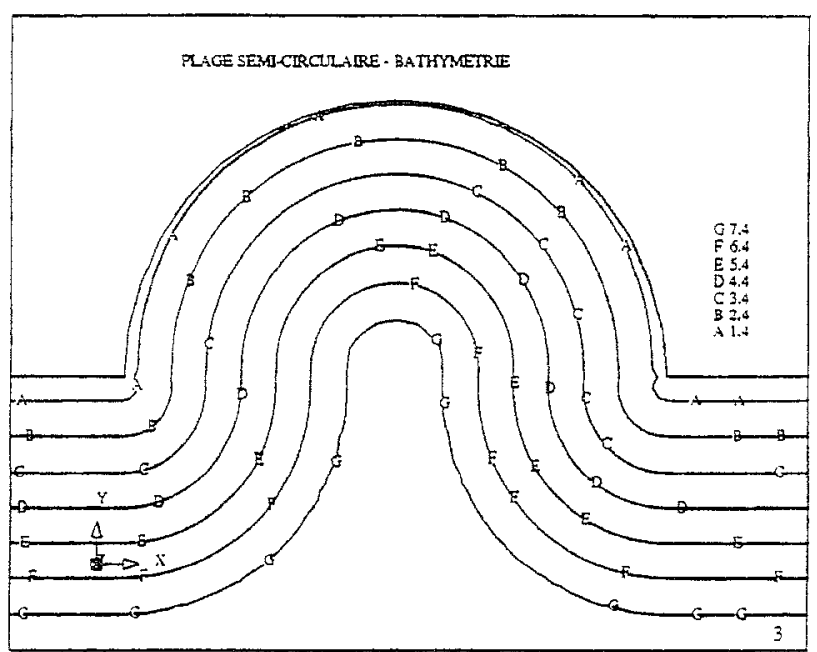

Figure 1: Domaine d'étude

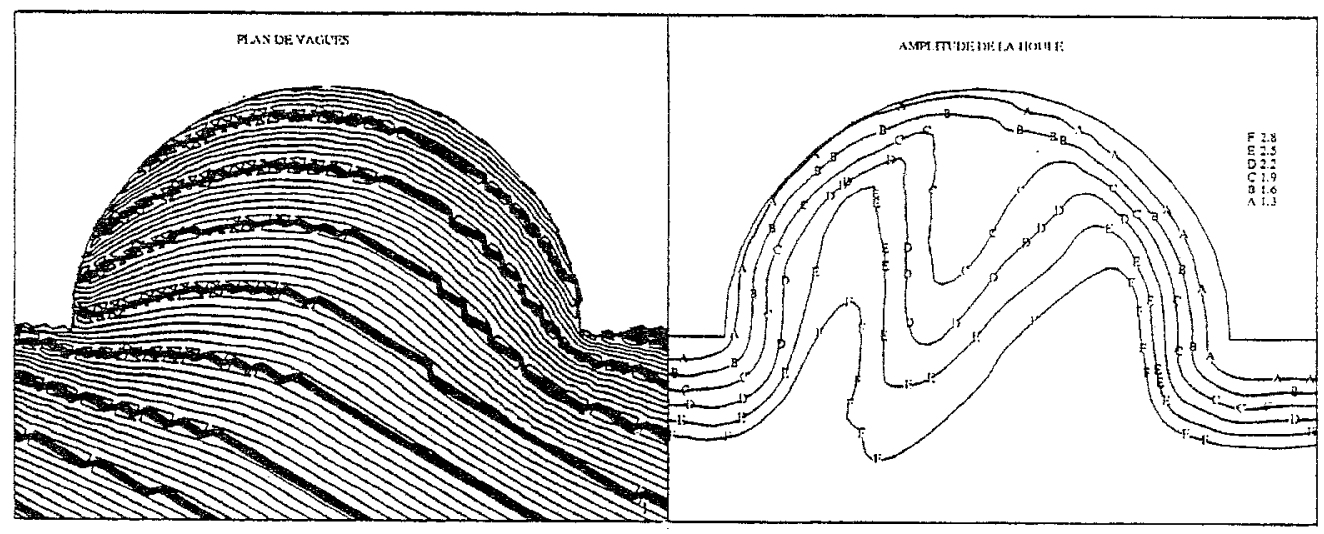

Figure 2: Champ de vagues et amplitude la houle

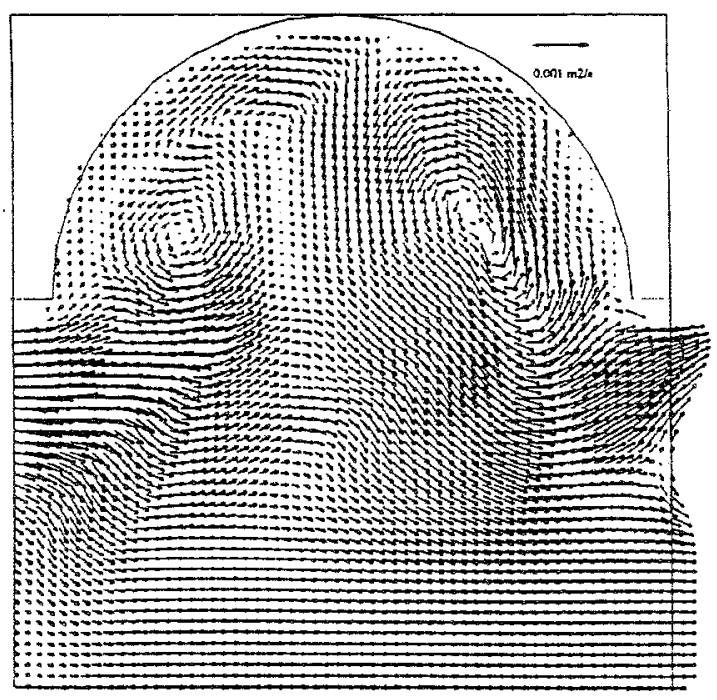

Figure 3: Transport solide 


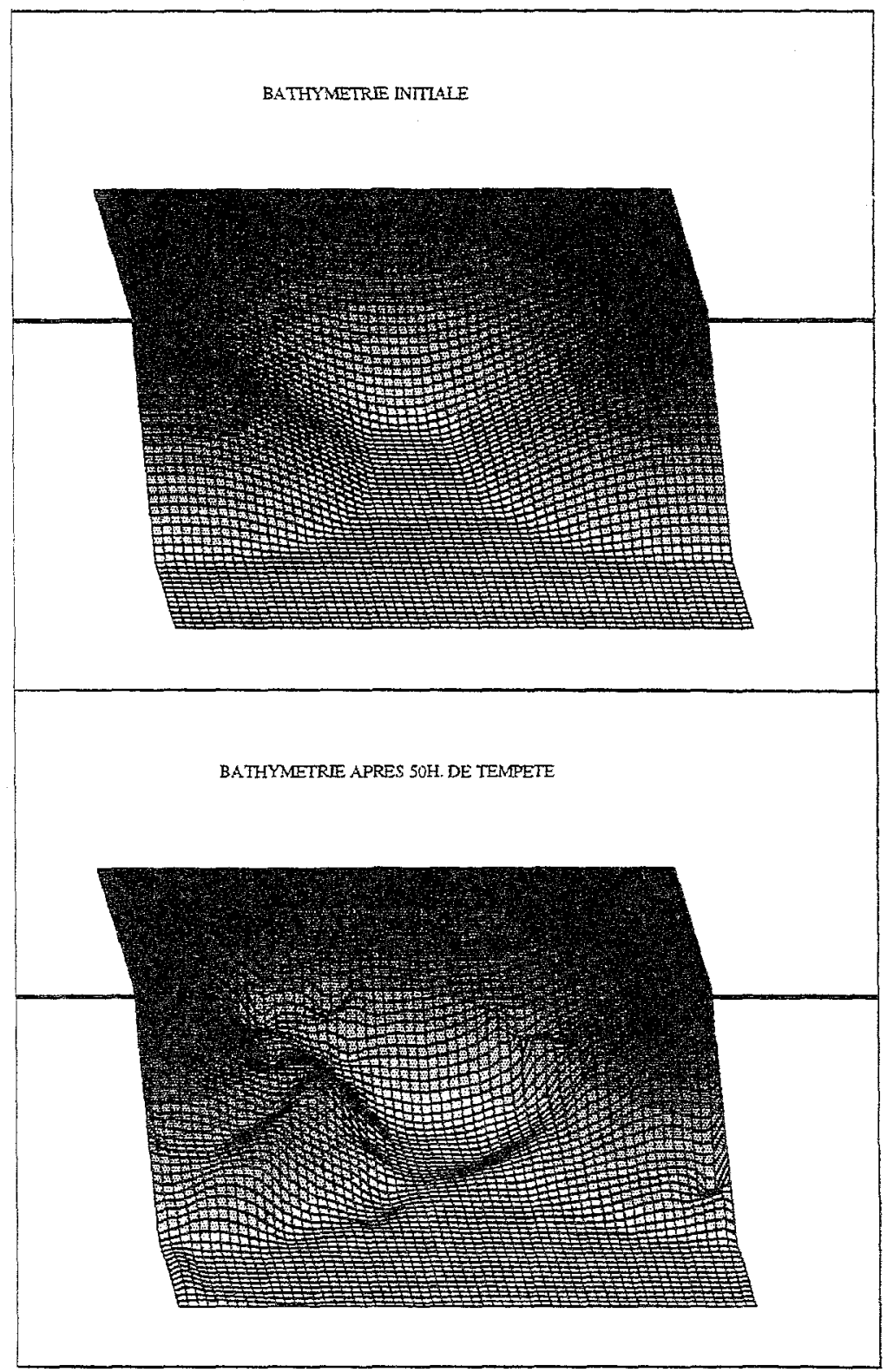

Figure 4: Evolution de la bathymétrie après $50 \mathrm{~h}$ de tempète 